\title{
La motivación como variable influyente en la ansiedad y autoconfianza de los deportistas
}

\section{Motivation as an influential variable in anxiety and self-confidence of athletes}

Fecha de recepción: 03/10/2016

Fecha de aceptación: 10/10/2016

\author{
Patricia Catalá Mesón* \\ Cecilia Peñacoba Puente* \\ Lilian Velasco Furlong* \\ Noelia Jareño Arias Correo* \\ Ángel Fernández Serrano* \\ *Facultad de Ciencias de la Salud. Universidad Rey Juan Carlos. Madrid
}

\section{resumen/ahstract:}

Diferentes trabajos analizan el vínculo que se establece entre el continuo de motivación y los niveles de ansiedad y autoconfianza en el ámbito deportivo. El propósito de este estudio es ahondar en esta relación. La muestra está formada por 562 jugadores de fútbol masculino pertenecientes a seis equipos de la comunidad de Madrid, con edades comprendidas entre los 12 y los 19 años. Una vez obtenido el consentimiento por parte de los entrenadores, padres y menores, se procedió a administrar, antes de una de sesiones de entrenamiento, el Cuestionario de Regulación Conductual en el Deporte (BRSO) y el Inventario de Ansiedad Estado Competitiva 2 (CSAI-2). Los resultados ponen de manifiesto que la regulación introyectada se muestra como la única variable predictora de la ansiedad cognitiva, la motivación intrínseca de estimulación y de conocimiento de la autoconfianza, y la motivación intrínseca de ejecución de la ansiedad somática (en este caso con relaciones negativas). Una baja desmotivación y una alta motivación intrínseca hacia la estimulación se muestran como variables esenciales a la hora de diferenciar perfiles positivos de futbolistas en relación a la ansiedad y autoconfianza en su conjunto. Se plantean líneas futuras de investigación, incluyendo el estudio de posibles variables moderadoras de la relación motivación-ansiedad, y la profundización en los procesos de actuación de la regulación introyectada.

Different studies analyze the link established between the continuum of motivation and anxiety and self-confidence in sports. The purpose of this study is to delve into this relationship. The sample consists of 562 male soccer players from six teams in the Community of Madrid, aged between 12 and 19 years. Once the informed consent was obtained by the coaches, parents and soccer players, we proceeded to administer, prior to a training session, the Behavioral Regulation in Sport Questionnaire (BRSO) and the Competitive Anxiety State Inventory 2 (CSAl- 2). The results show that the introjected regulation is shown as the only predictor of cognitive anxiety, intrinsic motivation to experience stimulation and intrinsic motivation to know are shown as predictors of self-confidence, and intrinsic motivation to accomplish things is shown as predictor of somatic anxiety (in this case with negative relationships). Both as a low a motivation such as a high intrinsic motivation to experience stimulation are essential to differentiating positive player profiles in relation to anxiety and self-confidence as a whole. Future research lines are proposed, including moderating variables of motivation-anxiety relationship and introjected regulation processes.

\section{palabras clave/keywords:}

Palabras clave: motivación, ansiedad, autoconfianza, deporte, futbolistas.

motivation, anxiety, self-confidence, sport, soccer players. 
En el contexto deportivo, la motivación ha despertado el interés de numerosas publicaciones (Clancy, Herring, MacIntyre y Campbell, 2016; Deaner, Balish y Lombardo, 2016; Irwin y Feltz, 2016), entendiéndose como un componente de influencia en el rendimiento deportivo (Gillet, Berjot, Vallerand, Amoura y Rosnet, 2012). El estudio de la motivación, y en particular de sus componentes intra (la capacidad de motivarnos internamente o automotivación) e interpersonales (la motivación a través de terceros), tiene importantes implicaciones en las prácticas de entrenamiento en los deportistas profesionales (Irwin y Feltz, 2016; Martinent, Decret, Guillet-Descas y Isoard-Gautheur, 2014; Rumpf, Schneider, Schneider y Mayer, 2014; van de Pol y Kavussanu, 2012).

La teoría de la motivación autodeterminada (Self-determination Theory; SDT) (Deci y Ryan, 1985) constituye un modelo explicativo de la misma, proporcionando respuestas sobre la involucración, más o menos libre, en la realización de actividades, teniendo en cuenta una serie de mecanismos psicológicos reguladores de la conducta. Desde esta aproximación conceptual, la motivación autodeterminada se corresponde con la motivación intrínseca, mientras que la motivación extrínseca favorece la conducta no autodeterminada e incluso caracterizada por la ausencia de motivación (Standage y Vallerand, 2014).

De manera específica, en el continuo establecido desde la SDT, según Moreno y Martínez (2006) la desmotivación hace referencia a la falta absoluta de motivación, ya sea intrínseca o extrínseca. La motivación extrínseca está determinada por recompensas externas, distinguiéndose además cuatro subtipos: a) la regulación externa (forma menos autodeterminada caracterizada por la búsqueda de recompensas y/o la evitación de castigo, b) la regulación introyectada, asociada con expectativas de auto-aprobación, evitación de la ansiedad y logro de mejoras del ego, c) la regulación identificada, que implica la percepción de valor e importancia de la conducta, por lo que se realizará la actividad voluntariamente aunque no sea agradable, y d) la regulación integrada, que conlleva conductas realizadas libremente en congruencia a los valores y necesidades.

La motivación intrínseca es la forma más autodeterminada del continuo, implicando voluntariedad y disfrute. Se realiza la actividad por el mero placer que se experimenta al practicarla; así, la práctica en si misma constituye el objetivo y la gratificación, suscitando sensaciones de competencia y autorealización. Se plantea a su vez la existencia de tres tipos de motivación intrínseca en función del objeto de obtención de la gratificación: a) hacia el conocimiento (el placer se experimenta por el hecho de aprender), b) hacia la ejecución (el placer se asocia a la superación personal), y c) hacia la estimulación (el placer se asocia a la obtención de nuevas sensaciones) (Standage y Vallerand, 2014).

Otra variable especialmente analizada en el contexto del rendimiento deportivo es la ansiedad (Carson y Collins, 2016; Mosconi, Correche, Rivarola y Penna, 2007; Uphill, 2016). Las numerosas publicaciones al respecto han abordado diferentes cuestiones como la elaboración y validación de instrumentos específicos para su medida en el ámbito deportivo (Rex y Metzler, 2016), el análisis de sus posibles predictores y la influencia de las variables sociodemográficas (Robinson y Freeston, 2015), o sus implicaciones en el rendimiento, la salud y el bienestar de los deportistas (Schaefer, Vella, Allen y Magee, 2016). 
El análisis de la posible relación entre las variables motivacionales anteriormente descritas y la ansiedad en el contexto deportivo puede aportar información relevante que facilite el diseño de programas de entrenamiento que potencien el rendimiento de los deportistas, garantizando al mismo tiempo la salud y el bienestar de los mismos (Carrasco, García-Mas y Brustad, 2009). Atendiendo a la literatura existente sobre ansiedad y motivación, puede observarse que gran parte de los trabajos se han centrado en el efecto que tienen estas variables, tomadas en su conjunto, sobre el rendimiento en deportistas de alta competición (Smrdu, 2015). Algunas investigaciones se han centrado especialmente en evaluar la relación existente entre la ansiedad y la motivación (Amado, Sánchez-Miguel, Leo, SánchezOliva y García-Calvo, 2013), encontrándose resultados no siempre coincidentes. Así, algunos trabajos no encuentran relación significativa entre motivación y ansiedad (Barber, Sukhi y White, 1999; Dureha, Singh, Yaduvanshi y Mishra, 2010). Otros, por el contrario, encuentran relaciones de distinta índole, como relaciones inversas entre la motivación por la tarea y la ansiedad (Williams, 1998), relaciones negativas entre el clima motivacional orientado al ego y los componentes cognitivo y somático de la ansiedad (Cervelló, Santos, Jiménez, Nerea y Gracia, 2002), relaciones positivas entre la desmotivación y la ansiedad cognitiva (Zarauz y Ruiz, 2015) o relaciones positivas entre la motivación extrínseca y ansiedad (López, 2011). Garcia-Mas et al. (2015) encontraron resultados diferenciados entre la motivación autodeterminada y la motivación de logro respecto a la ansiedad competitiva. Así, la motivación autodeterminada no mostraba ninguna influencia sobre la ansiedad, sin embargo dependía totalmente de los componentes de orientación a metas de ego y de tarea, tanto en la disposición de los jugadores como del clima motivacional percibido. Hanton, Wadey y Mellalieu (2008) ponen de manifiesto que un elevado control de la atención, el aumento de la motivación y el esfuerzo aumentan la percepción de control sobre los síntomas relacionados con la ansiedad.

Como puede observarse en los trabajos revisados, existe variabilidad respecto a los resultados obtenidos en la relación motivación y ansiedad, presentando además algunos de ellos ciertas limitaciones metodológicas, al tratarse en algunos casos de estudios correlacionales de pequeño tamaño muestral. Además, resulta complicado extraer conclusiones generalizables, debido a los diferentes marcos conceptuales empleados en el estudio de la motivación en el ámbito deportivo como la teoría de la necesidad de logro (Atkinson, 1974; McClelland, 1961), la teoría de la atribución (Weiner, 1986), la teoría de orientación de meta de logro (Duda, 1993) o la teoría de la motivación autodeterminada (Deci y Ryan, 1985), entre otros. En este contexto, y tal y como pone de manifiesto Ersöz (2016), resulta de especial interés analizar esta relación en el marco de la teoría de la motivación autodeterminada, por constituir, como se ha señalado, un marco de referencia de estudio en el contexto deportivo.

Es por ello que el principal objetivo de este estudio es analizar la relación existente entre el continuo de motivación, planteado desde la teoría de la motivación autodeterminada, y los niveles de ansiedad y autoconfianza en futbolistas españoles, habida cuenta de las características diferenciales de este grupo de deportistas (Belando et al., 2015). Nuestra hipótesis de trabajo plantea que existirán perfiles motivacionales diferenciales asociados a la ansiedad somática y cognitiva, y a la autoconfianza. Así, podría hipotetizarse que la moti- 
vación intrínseca se encontrará positivamente asociada a la autoconfianza de los jugadores, mientras que la motivación extrínseca se asociará de manera positiva a los niveles de ansiedad somática y cognitiva, esperando, de manera específica, una relación entre la regulación introyectada y la ansiedad cognitiva.

\section{Método}

\section{Participantes}

Participaron en el presente estudio 562 hombres jugadores de fútbol pertenecientes a seis equipos de la Comunidad de Madrid. Todos los participantes entrenaban entre dos y tres veces por semana (una hora y media por sesión de entrenamiento) y competían regularmente en fútbol federado a nivel regional y/o nacional. La edad media de los participantes era de 15.29 años $(\mathrm{DT}=1.86$, rango de edad $=12-19$ años $)$. Las categorías incluidas fueron infantil $(n=159,28.3 \%)$, cadete $(n=184,32.7 \%)$ y juvenil $(n=219,39 \%)$. Respecto al nivel de estudios, el 76.51\% ( $\mathrm{n}=430)$ de los participantes tienen estudios equivalentes a la ESO, el $22.24 \%(\mathrm{n}=125)$ estudios de bachillerato, y el $0.89 \%(\mathrm{n}=5)$ cuenta con estudios universitarios. Finalmente, el $0.36 \%(\mathrm{n}=2)$ no tiene estudios.

Todos los jugadores participaron de forma voluntaria y desinteresada, sin recibir ningún tipo de incentivo por su participación. Previamente a la administración de los cuestionarios, se obtuvo el consentimiento informado de entrenadores y padres, así como la autorización de estos últimos para la participación de los jugadores en el estudio.

\section{Instrumentos}

Cuestionario de regulación conductual en el Deporte (BRSQ) (Lonsdale, Hodge y Rose, 2008). Se empleó la versión traducida y validada por Moreno-Murcia, Marzo, MartínezGalindo y Marín (2011). La escala consta de un total de 36 ítems, con un formato de respuesta tipo Likert de siete puntos, que permiten medir las ocho dimensiones del continuo de motivación. Todas las afirmaciones son directas, y la puntuación total de cada subescala se obtiene promediando las respuestas en sus cuatro ítems, obteniendo por lo tanto un rango de puntuaciones teóricas entre 1 y 7 , de manera que una puntuación más elevada se interpreta como una mayor regulación del tipo que mide la subescala. En el estudio de Moreno-Murcia et al. (2011), se obtuvieron los siguientes valores de Alpha de Cronbach: .83 en desmotivación, .63 en regulación externa, .77 en regulación introyectada, .68 en regulación identificada, .78 en regulación integrada, .78 en motivación intrínseca hacia el conocimiento, .80 en motivación intrínseca hacia la ejecución y .78 en motivación intrínseca hacia la estimulación. En el presente estudio, los Alphas de Cronbach para cada una de las dimensiones fueron: .82 en desmotivación, .78 en regulación externa, .78 en regulación introyectada, .73 en regulación identificada, .77 regulación integrada, .75 en motivación intrínseca de conocimiento, .77 en motivación intrínseca de ejecución y .79 en motivación intrínseca de estimulación.

Inventario de ansiedad estado competitiva 2 (Competitive State Anxiety Inventory 2, CSAI2, Martens, Burton, Vealey, Bump y Smith, 1990). Se empleó la versión española de Capdevila (1997). Este cuestionario evalúa los componentes cognitivos y somáticos de la 
ansiedad estado y la autoconfianza en relación a la ejecución deportiva referida a la competición. Consta de 27 ítems, con un formato de respuesta tipo Likert de 4 puntos, que evalúan tres factores: ansiedad cognitiva (9 ítems), ansiedad somática (9 ítems) y autoconfianza ( 9 ítems). La puntuación total de cada dimensión se obtiene del promedio de las puntuaciones de sus ítems, oscilando, por lo tanto, el rango teórico de cada dimensión entre 1 y 4 , indicando mayores puntuaciones una mayor intensidad en la variable en cuestión. Capdevila (1997) encontró valores Alpha de Cronbach de .91 para ansiedad cognitiva, .93 para ansiedad somática y .90 para autoconfianza.En el presente estudio, los Alphas de Cronbach para cada una de las dimensiones fueron: .70 para ansiedad cognitiva, .76 para ansiedad somática y .82 para autoconfianza.

\section{Procedimiento}

Los datos fueron recogidos entre enero y mayo de 2014. Se establecieron reuniones con los coordinadores deportivos de los equipos para que informaran a los entrenadores y a los padres, obteniendo así el permiso de estos últimos para poder proceder a pasar los cuestionarios a los menores. Estos fueron entregados a los niños y adolescentes por uno de los investigadores antes del inicio del entrenamiento con el fin de evitar la fatiga o posibles respuestas sentimentales (p. e. después de un buen o mal entrenamiento).

La evaluación se llevó a cabo en salas acondicionadas y tuvo una duración de 40 minutos aproximadamente.

\section{Análisis de datos.}

Los análisis se realizaron con el programa estadístico SPSS 21 (Armonk, Nueva York, EE.UU.). Se llevaron a cabo análisis descriptivos y de consistencia interna (coeficiente alpha de Cronbach) de las dimensiones de motivación y de ansiedad, así como diferentes tipos de análisis para analizar la relación entre las variables sociodemográficas y las variables motivacionales, de ansiedad y autoconfianza, en función de la naturaleza de las primeras ( $\mathrm{t}$ de Student en el caso de los estudios, ANOVA en el caso de la categoría, correlación de Pearson en el caso de la edad). Se realizaron igualmente análisis de correlaciones de Pearson entre las variables de motivación, ansiedad y autoconfianza. Finalmente, se realizaron análisis de regresión lineal multivariada y análisis de regresión logística binaria con el objetivo de analizar el papel predictor de las modalidades de motivación en la ansiedad cognitiva y somática y la autoconfianzatanto de manera independiente como en su conjunto a través de una variable compuesta (perfil positivo). Estos últimos análisis aparecen detallados en los apartados correspondientes de resultados.

\section{Resultados}

\section{Análisis descriptivos.}

En la Tabla 1 se muestran los estadísticos descriptivos de las dimensiones de motivación y de ansiedad. Como puede observarse, los valores más altos de motivación se corresponden con las dimensiones más autodeterminadas, en particular la regulación integrada y las tres modalidades de motivación intrínseca, en todos los casos con valores medios superiores a 6 . 
Tabla 1. Análisis descriptivos.

\begin{tabular}{l|c|c|c|c}
\hline & Media (DT) & Asim. & Kurt. & PC50 \\
\hline 1. Desmotivación & $2.11(1,58)$ & 1.50 & 1.32 & 2.11 \\
2. Regulación externa & $2.35(1.53)$ & 1.08 & 0.29 & 1.75 \\
3. Regulación introyectada & $3,70(1.82)$ & 0.09 & -1.06 & 3.75 \\
4. Regulación identificada & $5.78(1.13)$ & -0.98 & 0.50 & 6.00 \\
5. Regulación integrada & $6.10(1.02)$ & -1.54 & 2.67 & 6.50 \\
6. Motiv. intrínseca de ejecución & $6,33(0.88)$ & -1.87 & 3.75 & 6.75 \\
7. Motiv. intrínseca de estimulación & $6.22(0.90)$ & -1.45 & 1.94 & 6.50 \\
8. Motiv. intrínseca de conocimiento & $6.00(1.00)$ & -1.25 & 1.64 & 6.25 \\
9. Ansiedad somática & $1.77(0.44)$ & 0.76 & 0.53 & 1.67 \\
10. Ansiedad cognitiva & $2.28(0.56)$ & 0.13 & -0.36 & 2.22 \\
11. Autoconfianza & $3.37(0.50)$ & -0.80 & 0.47 & 3.44 \\
\hline
\end{tabular}

$* \mathrm{p}<0.05 ; * * \mathrm{p}<0.01$

\section{Relación con las variables sociodemográficas}

Respecto a la relación de las variables de interés con las variables sociodemográficas consideradas (edad, categoría y nivel de estudios), pueden observarse relaciones positivas entre la edad y la motivación intrínseca de estimulación $\left(\mathrm{r}^{2}=.11 ; \mathrm{p}=.01\right)$ por un lado, y relaciones negativas entre la edad y la desmotivación $\left(\mathrm{r}^{2}=-.11 ; \mathrm{p}=.01\right)$ por otro.

En relación a la categoría, se observan diferencias estadísticamente significativas en la regulación identificada $(\mathrm{F}(2,545)=4.181, \mathrm{p}=.01)$ con mayores puntuaciones en la categoría de juvenil $(M=5.92, \mathrm{DT}=1.00)$ respecto a cadete $(\mathrm{M}=5.60, \mathrm{DT}=1.17)(\mathrm{p}=.02)$, en la motivación intrínseca de estimulación $(\mathrm{F}(2,545)=4.263, \mathrm{p}=.015)$ con mayores puntuaciones en juvenil $(\mathrm{M}=6.33$, DT $=0.78)$ que en cadete $(\mathrm{M}=6.08, \mathrm{DT}=1.00)(\mathrm{p}=.02)$, y en la motivación intrínseca de conocimiento $(\mathrm{F}(2,545)=4.021, \mathrm{p}=.018)$ con mayores puntuaciones en infantil $(\mathrm{M}=6.17, \mathrm{DT}=0.93)$ respecto a cadete $(\mathrm{M}=5.86, \mathrm{DT}=1.09)(\mathrm{p}=.02)$.

Dada la escasez de muestra con estudios universitarios $(0.89 \%)$ y sin estudios $(0.36 \%)$ se procedió a realizar un contraste de medias ( t de Student) entre los dos grupos más prevalentes en relación al nivel de estudios: ESO y Bachillerato. Los resultados ponen de manifiesto diferencias estadísticamente significativas en relación a la desmotivación $(\mathrm{t}(198,328)=2.360$, $\mathrm{p}=.02)$, presentando los jugadores con nivel educativo de ESO mayores puntuaciones $(\mathrm{M}=$ 2.18; DT=1.63) que los jugadores con nivel de Bachillerato $(\mathrm{M}=1.81$; $\mathrm{DT}=1.39)$. Se observan igualmente diferencias en relación a la motivación intrínseca hacia la estimulación ( $\mathrm{t}(195,449)=-2.105 ; \mathrm{p}=.037)$, observándose mayores puntuaciones en los jugadores con un nivel de Bachillerato $(\mathrm{M}=6.35$; $\mathrm{DT}=.79)$ que en los jugadores con un nivel de ESO $(\mathrm{M}=$ 6.17; DT=.91). 


\section{Correlaciones entre las variables de interés}

En cuanto a la correlación entre las variables de interés, tal y como se muestra en la Tabla 2, puede observarse que las motivaciones más autodeterminadas (motivación intrínseca de conocimiento, motivación intrínseca de estimulación, motivación intrínseca de ejecución y regulación integrada) correlacionan negativamente con la desmotivación, y positivamente entre sí. La ansiedad somática correlaciona negativamente con los tres tipos de motivación intrínseca. Además, ambos tipos de ansiedad correlacionan de forma positiva con la desmotivación. La autoconfianza es la variable en la que se observa un mayor número de correlaciones, positivas con las motivaciones más autodeterminadas y negativa con la desmotivación.

Tabla 2. Correlaciones entre las variables de interés.

\begin{tabular}{|c|c|c|c|c|c|c|c|c|c|c|}
\hline & 2 & 3 & 4 & 5 & 6 & 7 & 8 & 9 & 10 & 11 \\
\hline 1. Desmotivación & $.66 * *$ & $.42 * *$ & -.05 & $-.16 * *$ & $-.30 * *$ & $-.21 * *$ & $-.09 *$ & $.11 *$ & $.12 * *$ & $-.09 *$ \\
\hline 2. Regulación externa & & $.57 * *$ & $.31 * *$ & $-.10 * *$ & $-.17 * *$ & .02 & -.02 & .08 & .08 & -.03 \\
\hline 3. Regulación introyectada & & & $.27 * *$ & $.24 * *$ & $.22 * *$ & $.17 * *$ & $.11 *$ & .05 & $.12 * *$ & .02 \\
\hline 4. Regulación identificada & & & & $.62 * *$ & $.61 * *$ & $.60 * *$ & $.57 * *$ & $-.09 *$ & -.03 & $.22 * *$ \\
\hline 5. Regulación integrada & & & & & $.57 * *$ & $.73 * *$ & $.70 * *$ & -.06 & -.05 & $.26 * *$ \\
\hline 6. Motiv. intrínseca de ejecución & & & & & & $.79 * *$ & $.65 * *$ & $-.10 * *$ & -.04 & $.27 * *$ \\
\hline 7. Motiv. intrínseca de estimulación & & & & & & & $.78 * *$ & $-.09 *$ & .01 & $.29 * *$ \\
\hline 8. Motiv. intrínseca de conocimiento & & & & & & & & $-.14 * *$ & -.02 & $.28 * *$ \\
\hline 9. Ansiedad somática & & & & & & & & & $.49 * *$ & $-.40 * *$ \\
\hline 10. Ansiedad cognitiva & & & & & & & & & & $-.29 * *$ \\
\hline 11. Autoconfianza & & & & & & & & & & \\
\hline
\end{tabular}

$* \mathrm{p}<0.05 ; * * \mathrm{p}<0.01$

\section{Predictores motivacionales de la ansiedad y autoconfianza deportiva}

Con el fin de analizar el valor predictivo de los diferentes tipos de motivación sobre la ansiedad y la autoconfianza, se llevaron a cabo tres análisis de regresión lineal multivariada por pasos sucesivos tomando como variable a predecir la ansiedad somática, la ansiedad cognitiva y la autoconfianza en cada uno de los casos. Para cada una de las ecuaciones se incluyeron como variables predictoras, en un primer paso las variables sociodemográficas de interés (edad, categoría y nivel de estudios) con el fin de controlar el efecto de las mismas, procediendo en el caso de la categoría y el nivel de estudios a su recodificación en variables dummy; en un segundo paso, se incluyeron las ocho modalidades de motivación.

Por favor, Insertar Tabla 3 
Tabla 3. Análisis de regresión: motivación, ansiedad y autoconfianza

\begin{tabular}{|c|c|c|c|c|}
\hline Dimensiones & $\mathrm{F}$ & $\mathrm{R} 2$ & Beta & $\mathrm{t}$ \\
\hline \multicolumn{5}{|l|}{ Ansiedad cognitiva } \\
\hline Paso 1: Edad & & .007 & -.100 & $-2.318^{*}$ \\
\hline Paso 2: Reg. introyectada & $6.236^{* *}$ & .019 & .121 & $2.803 * *$ \\
\hline \multicolumn{5}{|l|}{ Ansiedad sómatica } \\
\hline Paso 1: Motiv. intrinseca de ejecución & $12.325^{* *}$ & .023 & -.151 & $-3.511 * *$ \\
\hline \multicolumn{5}{|l|}{ Autoconfianza } \\
\hline \multicolumn{5}{|l|}{ Paso 1: Motiv. intrinseca de estimulación } \\
\hline \multirow[t]{2}{*}{ Paso 2: Motiv. intrinseca de conocimiento } & & .090 & .233 & $4.372 * *$ \\
\hline & $28.974 * *$ & .095 & .109 & $2.047^{*}$ \\
\hline
\end{tabular}

$* \mathrm{p}<0.05 ; * * \mathrm{p}<0.01$

Como puede observarse en la Tabla 3, la regulación introyectada se muestra como la única variable predictora de la ansiedad cognitiva, contribuyendo a la explicación de un $1.9 \%$ de la varianza. La motivación intrínseca de ejecución se muestra (negativamente) como predictora de la ansiedad somática, contribuyendo a un $2.3 \%$ de la varianza, y finalmente, la motivación intrínseca de estimulación y la motivación intrínseca de conocimiento contribuyen a la explicación de la autoconfianza (9.5\% de la varianza). La edad únicamente contribuye de manera significativa y negativa a la ansiedad cognitiva.

Variables motivacionales en la diferenciación de perfiles positivos respecto a la ansiedad y la autoconfianza

Con el objetivo de tener una medida compuesta que relacionara las tres variables (ansiedad somática, ansiedad cognitiva y autoconfianza), se planteó el empleo de una variable compuesta que empleara como criterio normativo la puntuación correspondiente al PC75 para las tres variables. Así, se consideró como perfil positivo en relación a la ansiedad y autoconfianza la concurrencia de 3 criterios: 1) Puntuaciones iguales o superiores al PC75 en la variable autoconfianza (estableciéndose el punto de corte en 3.66),2) Puntuaciones inferiores al PC75 en la variable ansiedad somática (estableciéndose el punto de corte en 1.89), 3) Puntuaciones inferiores al PC75 en la variable ansiedad cognitiva (estableciéndose el punto de corte en 2.63). Como consecuencia de esta clasificación, obtenemos un 19.6\% $(n=110)$ de jugadores con perfil positivo, frente a un $80.4 \%(n=452)$ de jugadores que no reunirían dicho perfil.

Se llevó a cabo una regresión logística binaria con el objetivo de predecir la presencia/ ausencia de perfil positivo tal y como ha sido definido previamente. Se incluyeron, como 
predictores, la edad como variable sociodemográfica de interés, así como las ocho modalidades de motivación objeto de estudio. Se realizaron análisis exploratorios utilizando regresiones logísticas para cada variable. El criterio para la inclusión de las variables en el análisis multivariado posterior fue establecido en valores de $\mathrm{p}$ (Wald) $<0.20$ en las regresiones univariadas (Hosmer y Lemeshow, 2000). Los predictores que cumplieron los criterios se introdujeron en el análisis multivariado mediante el método BSTEP (LR), aplicándose igualmente la prueba de ajuste de Hosmer y Lemeshow.

Los análisis univariados previos ponen de manifiesto los siguientes predictores: desmotivación $(\mathrm{OR}=.815, \mathrm{p}=.013)$, regulación identificada $(\mathrm{OR}=1.434, \mathrm{p}=.001)$, regulación integrada $(\mathrm{OR}=1.697, \mathrm{p}<.000)$, motivación intrínseca hacia el conocimiento $(\mathrm{OR}=1.555$, $\mathrm{p}=.001)$, motivación intrínseca hacia la estimulación ( $\mathrm{OR}=2.151, \mathrm{p}<.000)$, y motivación intrínseca hacia la ejecución $(\mathrm{OR}=2.251, \mathrm{p}<.000)$. Los resultados de la regresión logística multivariada señalan que los jugadores que presentan ese perfil positivo, comparado con aquellos que no lo presentan, tienen una menor probabilidad de presentar desmotivación $(\mathrm{OR}=.877 ; 95 \% \mathrm{CI}=.749,1.026)$ y una mayor probabilidad de presentar motivación intrínseca hacia la estimulación $(\mathrm{OR}=2.057 ; 95 \% \mathrm{CI}=1.445,2.929)$ en comparación con el resto de jugadores $(\chi 2=27.394, \mathrm{p}<.000$; Goodfit test: $\mathrm{p}=.397)$.

\section{Discusión}

El presente artículo tiene como principal objetivo analizar las diferentes dimensiones motivacionales en una muestra de futbolistas españoles así como su relación con la ansiedad competitiva y la autoconfianza. Los resultados ponen de manifiesto altos niveles de motivación intrínseca y bajos niveles de motivación externa y desmotivación, en coherencia con estudios previos realizados en diferentes muestras de deportistas (Zarauz y Ruíz, 2015) y en particular en futbolistas (Belando et al., 2015).

Respecto a las variables sociodemográficas, la edad se asocia a una mayor motivación intrínseca de estimulación y a menores niveles de desmotivación. Los datos encontrados en la literatura a este respecto no son siempre coincidentes, teniendo en cuenta además la diversidad de la propia edad de las muestras y de los contextos deportivos analizados. Así, mientras algunos autores (Moreno-Murcia, Cervelló y González-Cutre, 2006) ponen de manifiesto una mayor motivación tanto intrínseca como extrínseca en los jugadores más jóvenes, y unos porcentajes mayores de desmotivación en los jugadores de mayor edad (Wang y Biddle, 2001), otros trabajos como el de Cuddihy y Corbin (1995) señalan, en línea con nuestros resultados, que la motivación intrínseca hacia la actividad deportiva se incrementa con la edad. La literatura precedente no parece aportar demasiados datos acerca de las relaciones entre motivación y categoría y nivel educativo, encontrándose en general, como en nuestro trabajo, mayor motivación intrínseca en niveles educativos más altos (Prieto, 2016) y en categorías más profesionales (de Oliveira et al., 2016). No obstante, los resultados encontrados en relación a estas variables en nuestra muestra en particular podrían ser debidos en parte al afecto de la edad, dado que en términos generales los resultados apuntan a mayores niveles de motivación intrínseca conforme se incrementa el nivel educativo y la categoría. Por otro lado, la edad se muestra como variable predictora de la ansiedad cogni- 
tiva, disminuyendo ésta cuando aumenta la edad del futbolista, en la línea de los resultados encontrados por Müller, Claes, Smits, Brähler y de Zwaan (2016).

Uno de los resultados de especial relevancia del presente trabajo es la aportación diferencial de las diferentes modalidades de motivación a la ansiedad y a la autoconfianza, poniendo de manifiesto, como se viene señalando en otras áreas de la psicología de la salud, que los predictores de la enfermedad no son los mismos que los del bienestar psicológico. Estos datos han sido encontrados igualmente en otros trabajos en el contexto deportivo, como el de Amado et al. (2013) que señala la motivación intrínseca como predictor positivo del flow disposicional y, en menor medida, de la ansiedad, mientras que la desmotivación se mostraba únicamente predictora de la ansiedad.

La relación entre motivación externa y ansiedad en el contexto deportivo ha sido estudiada bien de manera directa (López, 2011; Zarauz y Ruiz, 2014) o indirectamente a través de variables como la lesión deportiva. Prieto, Ortega, de los Fayos y Olmedilla (2014) aluden a un perfil de vulnerabilidad a la lesión (menor personalidad resistente, mayor ansiedad competitiva, menor motivación orientada al éxito y mayor motivación orientada a evitar el fracaso) no encontrando resultados concluyentes respecto a la predicción de la lesión, lo que indica la complejidad de los efectos de los niveles motivacionales. Otros trabajos han puesto de manifiesto un papel facilitador de la ansiedad, junto con el compromiso a correr y la percepción de éxito en las carreras como motivos principales para llevarlas a cabo (Zarauz, Ruiz, Arbinaga, Janes y Flores-Allende, 2015). Por otro lado, otros trabajos han relacionado igualmente la desmotivación con la ansiedad cognitiva (Zarauz y Ruiz, 2015), y con la ansiedad en general (Amado et al., 2013). Nuestros resultados ponen de manifiesto, en coherencia con los trabajos anteriores, correlaciones positivas entre la ansiedad, especialmente la cognitiva, y la regulación externa y la desmotivación. Sin embargo, los análisis de regresión muestran bajos niveles de motivación intrinseca de ejecución como predictores de la ansiedad somática.

Igualmente, resulta de especial interés las diferencias, en cuanto a su relación con las modalidades de motivación, entre la ansiedad somática y la ansiedad cognitiva. Así, nuestros resultados muestran que la ansiedad somática se asocia con menores niveles de motivación intrinseca de ejecución mientras que la ansiedad cognitiva se relaciona con mayores niveles de regulación introyectada. Aunque algunos de los estudios precedentes no encuentran resultados diferenciales en la comparación de las variables influyentes entre ansiedad somática y cognitiva (Cecchini, González, Carmona y Contreras, 2004), otros trabajos como el de Garcia-Mas et al. (2015) apuntan a estas diferencias motivacionales, encontrando distinto peso causal de la motivación autodeterminada y de la motivación de logro sobre la ansiedad competitiva, evidenciando una controvertida relación entre los climas motivacionales percibidos y las orientaciones de tarea y de ego de los jugadores, confirmando igualmente el papel residual de la ansiedad somática. Sancho y Ruiz (2014) encuentran igualmente predictores diferenciales entre la ansiedad somática y la cognitiva. Así, tanto una alta orientación a la tarea como una alta competencia percibida predecían bajos niveles de ansiedad cognitiva, mientras que los atletas con una alta competencia percibida presentaban menor ansiedad somática respecto a los de baja competencia percibida. 
En este contexto diferencial, el hecho de que la ansiedad somática se asocie negativamente a la motivación intrínseca hacia la ejecución parece verse apoyado por estudios previos como el de Buceta, López, Peréz, Vallejo y Del Pino (2003), quién encontró correlaciones negativas entre la ansiedad somática, la edad y la experiencia de los participantes.

Respecto a la ansiedad cognitiva, nuestros datos ponen de manifiesto que se relaciona positivamente con la regulación introyectada. Estos resultados podrían justificarse si tenemos en cuenta que la interiorización de las propias normas, la necesidad de auto-aprobación y de reconocimiento social, las presiones internas y los sentimientos de culpa, motores fundamentales de este tipo de regulación, suelen asociarse a procesos rumiativos y en consecuencia a niveles elevados de ansiedad cognitiva (Merino, Senra y Ferreiro, 2016). Fuera del ámbito deportivo, Chu (2015) encuentra apoyo a esta hipótesis, poniendo de manifiesto además que la cultura tiene un efecto moderador entre la autorregulación mediante la retención de las emociones negativas que se produce a través de la regulación introyectada y sus posibles efectos en la salud mental.

Dentro del contexto deportivo, los resultados sobre el papel de la regulación introyectada son escasos y contradictorios. En línea con nuestros resultados, Sicilia, Sáenz-Álvarez, González-Cutre y Ferriz (2014), ponen de manifiesto que la regulación introyectada tiene un efecto sobre la ansiedad física y social, tanto directo como mediador sobre el efecto en la misma de la necesidad de competencia. Igualmente, Winch, Moberly y Dickson (2015) ponen de manifiesto la relación diferencial de los diferentes tipos de motivación con la ansiedad y la depresión. En particular, los síntomas depresivos se asociaron únicamente a la motivación intrínseca vinculada a metas de aproximación (pero no de evitación), mientras que los síntomas de ansiedad se asociaron a la regulación introyectada vinculada a metas tanto de aproximación como de evitación.

No obstante, la literatura también arroja datos contradictorios respecto a los encontrados en nuestro trabajo como el puesto de manifiesto por Ersöz (2016) quien señala que el género y la motivación intrínseca e introyectada constituyen predictores positivos de la experiencia de flow, mostrándose la regulación externa como predictor positivo de la ansiedad física y social.

Aunque la mayor parte de los trabajos en el contexto deportivo se han centrado en el estudio de la ansiedad, algunos analizan igualmente la autoconfianza de los deportistas. Maugendre y Spitz (2011) ponen de manifiesto que la motivación autodeterminada se relaciona con las conductas adaptativas y con el bienestar psicológico en parte gracias al planteamiento de metas relacionadas con el desarrollo personal. Fülöp (2009) señala que la felicidad y las emociones positivas asociadas a la misma provienen en gran parte de la capacidad del deportista de vincular a las situaciones de fracaso consecuencias positivas como el aprendizaje acerca de uno mismo, tomar conciencia de las fortalezas y debilidades e incrementar la motivación para situaciones futuras. Estos resultados de trabajos previos son coherentes con nuestros resultados que apuntan el papel de la motivación intrínseca (estimulación y conocimiento) como predictores de la autoconfianza.

El presente trabajo cuenta con algunas limitaciones que conviene señalar, entre ellas la naturaleza de la muestra, que aunque amplia en número, es en su totalidad masculina y repre- 
senta un solo deporte en una zona geográfica determinada (Comunidad de Madrid). Los datos obtenidos por tanto permiten obtener una visión de la situación de los jóvenes futbolistas, pero su generalización a otras prácticas deportivas debería realizarse con cautela. Por otro lado, la naturaleza correlacional del estudio impide el establecimiento de relaciones causa-efecto, por lo que sería de interés en líneas futuras el empleo de estudios longitudinales. A pesar de las anteriores limitaciones, los resultados obtenidos ponen de manifiesto que los niveles de autoconfianza de los futbolistas se relacionan directamente con la motivación intrínseca tanto de estimulación como de conocimiento, mientras que los predictores de la ansiedad se asocian a la ausencia de motivación intrínseca de ejecución (en el caso de la somática) y a la presencia de regulación introyectada (en el caso de la ansiedad cognitiva). Tal y como señalan Mosconi, Correche, Rivarola y Penna (2007), la ansiedad y la motivación están consideradas como causas principales que influyen en el rendimiento, por lo que resulta de interés seguir investigando en este ámbito. En este sentido, y como futuras líneas de investigación, teniendo en cuenta que los porcentajes de varianza explicados no son considerablemente elevados (salvo en el caso de la autoconfianza), debería profundizarse en el papel de otras variables influyentes en la ansiedad competitiva, en su efecto directo o mediador, así como en los procedimientos de actuación de la regulación introyectada, sobre los que, desde nuestro punto de vista, queda mucho por explorar. La profundización en la línea de investigación iniciada en este trabajo tiene importantes repercusiones prácticas en el diseño de programas de intervención focalizados sobre las variables motivacionales y sus posibles determinantes con el objetivo de mejorar el rendimiento y la calidad de vida de los deportistas (Calvo, Pujals, Barragán y Calvo, 2012; Carrasco et al., 2009).

\section{Referencias}

Amado, D., Sánchez-Miguel, P. A., Leo, F. M., Sánchez-Oliva, D., y García-Calvo, T. (2013). Desarrollo de un modelo de motivación para explicar el flow disposicional y la ansiedad en bailarines profesionales. Universitas Psychologica, 12(2), 457-470. doi:10.11144/Javeriana.UPSY12-2.dmmf

Atkinson, J. W. (1974). The mainstream of achievement-oriented activity. En J. W. Atkinson y J. 0. Raynor (eds.). Motivation and achievement. New York. Halstead.

Barber, H., Sukhi, H., y White, S. A. (1999). The influence of parent-coaches on participant motivation and competitive anxiety in youth sport participants. Journal Of Sport Behavior, 22(2), 162-180.

Belando, N., Ferriz-Morel, R., Rivas, S., Almagro, B., Sáenz-López, P., Cervelló, E., y Moreno-Murcia, J. A. (2015). Sport commitment in adolescent soccer players. Motricidade, 11(4), 3-14. doi:10.6063/motricidade.2969

Buceta, J., López de la Llave, A., Peréz, M., Vallejo, M., y Del Pino, M. (2003). Estado psicológico de los corredores populares de maratón en días anteriores a la prueba. Psicothema, 15(2), 273-277.

Calvo, J. L., Pujals, C., Barragán, R. N., y Calvo, A. L. (2012). Análisis de los efectos de un programa de intervención psicológica en jóvenes jugadores de baloncesto. Revista de Psicología del deporte, 21(1), 43-48.

Capdevila, L. (1997). Metodología de evaluación en psicología del deporte. Psicología del Deporte. Madrid: Síntesis.

Carrasco, A. E. R., García-Mas, A., y Brustad, R. J. (2009). Estado del arte, y perspectiva actual del concepto de bienestar psicológico en psicología del deporte. Revista Latinoamericana de Psicología, 41(2), 335-347.

Carson, H. J., y Collins, D. (2016). The fourth dimension: A motoric perspective on the anxiety-performance relationship. International Review Of Sport And Exercise Psychology, 9(1), 1-21. doi:10.1080/1750984X.2015.1072231 
Cecchini, J. A., González, C., Carmona, Á. M., y Contreras, 0. (2004). Relaciones entre clima motivacional, la orientación de meta, la motivación intrínseca, la auto-confianza, la ansiedad y el estado de ánimo en jóvenes deportistas. = Relationships among motivational climate, achievement goals, intrinsic motivation, self-confidence, anxiety, and mood in young sport players. Psicothema, 16(1), 104-109.

Cervello , E., Santos, R., Jiménez, R., Nerea, A., y García, T. (2002). Motivación y ansiedad en jugadores de Tenis. Revista de motricidad, 9, 141-161.

Chu, L. (2015). The influence of the internalization of emotional regulation on mental health among the Taiwanese people: the moderating effect of cultural fit. Asia-Pacific Journal Of Public Health / Asia-Pacific Academic Consortium For Public Health, 27(2), NP1918-NP1931. doi:10.1177/1010539512455045

Clancy, R. B., Herring, M. P., MacIntyre, T. E., y Campbell, M. J. (2016). A review of competitive sport motivation research. Psychology Of Sport And Exercise, 27232-242. doi:10.1016/j.psychsport.2016.09.003

Cuddihy, T. F., y Corbin, C. B. (1995). Gender differences in intrinsic motivation toward physical activity in a high school population [Abstract]. Research Quarterly for Exercise and Sport, 66, A-30.

Deaner, R. 0., Balish, S. M., y Lombardo, M. P. (2016). Sex differences in sports interest and motivation: An evolutionary perspective. Evolutionary Behavioral Sciences, 10(2), 73-97. doi:10.1037/ebs0000049

De Oliveira, L. P., Do Nascimento Junior, J. A., Nickenig Vissoci, J. R., Ferreira, L., da Silva, P. N., y Lopes Vieira, J. L. (2016). Motivación autodeterminada y estrategias de afrontamiento en futbolistas: Un estudio con jugadores en diferentes fases de desarrollo deportivo. Revista De Psicología Del Deporte, 25(2), 261-269.

Deci, E. L., y Ryan, R. M. (1985).The general causality orientations scale: Self- determination in personality. Journal of Research in Personality, 19, 109-134.

Duda, J. L. (1993). Goals: A social-cognitive approach to the study of achievement motivation in sport. En R. N. Singer M. Murphey y L. K. Tennant (eds.). Handbook of research on sport psychology. New York. Macmillan.

Dureha, D. K., Singh, M., Yaduvanshi, S., y Mishra, P. (2010). A comparative study of incentive motivation, achievement motivation and anxiety level between national and international hockey players. British Journal Of Sports Medicine, 44(S1), i58.

Ersöz, G. (2016). An examination of motivational regulations, dispositional flow and social physique anxiety among college students for exercise: a self-determination theory approach. College Student Journal, 5O(2), 159-170.

Fülöp, M. (2009). Happy and Unhappy Competitors: What Makes the Difference?. Psihologijske Teme / Psychological Topics, 18(2), 345-367

García-Mas, A., Fuster-Parra, P., Ponseti, F., Palou, P., Olmedilla, A., y Cruz, J. (2015). Análisis de las relaciones entre la motivación, el clima motivacional y la ansiedad competitiva en jóvenes jugadores de equipo mediante una red Bayesiana. Anales De Psicología, 31(1), 355-366. doi:10.6018/analesps.31.1.167531

Gillet, N., Berjot, S., Vallerand, R. J., Amoura, S., y Rosnet, E. (2012). Examining the motivation-performance relationship in competitive sport: A cluster-analytic approach. International Journal Of Sport Psychology, 43(2), 79-102.

Hanton, S., Wadey, R., y Mellalieu, S. D. (2008). Advanced Psychological Strategies and Anxiety Responses in Sport. Sport Psychologist, 22(4), 472-490.

Hosmer, D. W., y Lemeshow, S. (2000). Introduction to the logistic regression model. Applied Logistic Regression, Second Edition, 1-30.

Irwin, B. C., y Feltz, D. L. (2016). Motivation gains in sport and exercise groups. In R. J. Schinke, K. R. McGannon, B. Smith, R. J. Schinke, K. R. McGannon, B. Smith (Eds.) , Routledge international handbook of sport psychology (pp. 494-504). New York, NY, US: Routledge/Taylor \& Francis Group.

Lonsdale, C., Hodge, K., y Rose, E. A. (2008). The behavioral regulation in sport questionnaire (BRSQ): Instrument development and initial validity evidence. Journal Of Sport \& Exercise Psychology, 30(3), 323-355.

López, N. I. (2011). Relación entre factores motivacionales y niveles de ansiedad en porristas en precompetencia. International Journal Of Psychological Research, 4(1), 80-91.

Martens, R., Burton, D., Vealey, R. S., Bump, L. A. y Smith, D. E. (1990). Development and validation of the competitive state 
anxiety inventory-2. Competitive anxiety in sport, 117-190.

Martinent, G., Decret, J., Guillet-Descas, E., y Isoard-Gautheur, S. (2014). A reciprocal effects model of the temporal ordering of motivation and burnout among youth table tennis players in intensive training settings. Journal Of Sports Sciences, 32(17), 1648-1658. doi:10.1080/02640414.2014.912757

Maugendre, M., y Spitz, E. (2011). Santé perçue, anxiété et motivation sportive. Annales Medico Psychologiques, 169(5)، 302-308. doi:10.1016/j.amp.2009.09.023

McClelland, D. (1961). The achieving society. New York. Free Press.

Merino, H., Senra, C., y Ferreiro, F. (2016). Are Worry and Rumination Specific Pathways Linking Neuroticism and Symptoms of Anxiety and Depression in Patients with Generalized Anxiety Disorder, Major Depressive Disorder and Mixed Anxiety-Depressive Disorder?. Plos 0NE, 11(5), 1-14. doi:10.1371/journal.pone.0156169

Moreno, J. A., \& Martínez, A. (2006). Importancia de la Teoría de la Autodeterminación en la práctica físico-deportiva: Fundamentos e implicaciones prácticas. Cuadernos de psicología del deporte, 6(2).

Moreno-Murcia, J. A., Cervello, E., y Gonzalez-Cutre, D. (2006). Motivación autodeterminada y flujo disposicional en el deporte. Anales de psicología, 22(2), 310-317.

Moreno-Murcia, J. A., Marzo, J. C., Martínez-Galindo, C., y Marín, L. C. (2011). Validación de la Escala de “Satisfacción de las Necesidades Psicológicas Básicas" y del Cuestionario de la "Regulación Conductual en el Deporte" al contexto español.(Validation of Psychological Need Satisfaction in Exercise Scale and the Behavioural Regulation in Sport Questionnaire to the Spanish context). RICYDE. Revista Internacional de Ciencias del Deporte, 7(26), 355-369. doi: 10.5232/ricyde,

Mosconi, S., Correche, M., Rivarola, M., y Penna, F. (2007). Aplicación de la técnica de relajación en deportistas de 16 años para mejorar su rendimiento. Fundamentos en Humanidades, 8 (16), p. 183-197.

Müller, A., Claes, L., Smits, D., Brähler, E., y de Zwaan, M. (2016). Prevalence and Correlates of Self-Harm in the German General Population. Plos ONE, 11(6), 1-17. doi:10.1371/journal.pone.0157928

Prieto, J. M. (2016). Relación entre competitividad, ansiedad social y compromiso con variables deportivas y académicas en futbolistas jóvenes. Revista Iberoamericana de Psicología del Ejercicio y el Deporte, 11(2), 193200.

Prieto, J. M., Ortega, E., de los Fayos, E. G., y Olmedilla, A. (2014). Perfiles de personalidad relacionados con la vulnerabilidad del deportista a lesionarse. Revista de Psicología del Deporte, 23(2), 431-437.

Rex, C. C., y Metzler, J. N. (2016). Development of the Sport Injury Anxiety Scale. Physical Education And Exercise Science, 20(3), 146-158. doi:10.1080/1091367X.2016.1188818

Robinson, G., y Freeston, M. (2015). Intolerance of uncertainty as a predictor of performance anxiety and robustness of sport confidence in university student-athletes. Journal Of Clinical Sport Psychology, 9(4), 335-344. doi:10.1123/jcsp.2015-0008

Rumpf, M. C., Schneider, A. S., Schneider, C., y Mayer, H. M. (2014). Training profiles and motivation of male and female youth soccer players. International Journal Of Sports Science \& Coaching, 9(1), 207-216. doi:10.1260/1747-9541.9.1.207

Sancho, A. Z., y Ruiz-Juan, F. (2014). Factores determinantes de la ansiedad en atletas veteranos españoles. = Determinants of anxiety in Spanish master athletes. Universitas Psychologica, 13(3), 1047-1058.

Schaefer, J., Vella, S. A., Allen, M. S., y Magee, C. A. (2016). Competition anxiety, motivation, and mental toughness in golf. Journal Of Applied Sport Psychology, 28(3), 309-320. doi:10.1080/10413200.2016.1162219

Sicilia, A., Sáenz-Alvarez, P., González-Cutre, D., y Ferriz, R. (2014). Exercise motivation and social physique anxiety in adolescents. Psychologica Belgica, 54(1), 111-129. doi:10.5334/pb.ai

Smrdu, M. (2015). First-person experience of optimal sport competition performance of elite team athletes. Kinesiology, 47(2), 169-178)

Standage, M., y Vallerand, R. J. (2014). Motivation in sport and exercise groups: A self-determination theory perspective. In M. R. Beauchamp, M. A. Eys, M. R. Beauchamp, M. A. Eys (Eds.) , Group dynamics in exercise and sport psychology, 2nd ed (pp. 259-278). New York, NY, US: Routledge/Taylor \& Francis Group. 
Uphill, M. (2016). Anxiety in sport: Are we any closer to untangling the knots?. In A. M. Lane, A. M. Lane (Eds.) , Sport and exercise psychology, 2nd ed (pp. 50-75). New York, NY, US: Routledge/Taylor \& Francis Group.

van de Pol, P. C., \& Kavussanu, M. (2012). Achievement motivation across training and competition in individual and team sports. Sport, Exercise, And Performance Psychology, 1(2), 91-105. doi:10.1037/a0025967

Weiner, B. (1986). An attribution theory of motivation and emotion. New York. Springer-Verlag.

Winch, A., Moberly, N. J., y Dickson, J. M. (2015). Unique associations between anxiety, depression and motives for approach and avoidance goal pursuit. Cognition \& Emotion, 29(7), 12951305. doi:10.1080/02699931.2014.976544

Wang, C. K. J., y Biddle, S. J. H. (2001). Young people's motivational pro- files in physical activity: A cluster analysis. Journal of Sport and Exercise Psychology, 23, 1-22.

Williams, L. (1998). Contextual influences and goal perspectives among female youth sport participants. Research Quarterly for Exercise and Sport, 69, p. 47-57.

Zarauz, A., y Ruiz, F. (2014). Factores determinantes de la ansiedad en atletas veteranos españoles. Universitas Psychologica, 13(3), 1047-1057. doi:10.11144/Javeriana.UPSY13-3.fdaa

Zarauz, A., y Ruiz, F. (2015). Factores determinantes de la motivación en atletas veteranos españoles. Revista Latinoamericana De Psicología, 47(1), 34-42.

Zarauz, A., Ruiz, F., Arbinaga, F., Jaenes, J. C., y Flores-Allende, G. (2015). Modelo predictor de las motivaciones para correr: análisis con corredores de ruta españoles vs. mexicanos. Universitas Psychologica, 14(2), 659-673. doi:10.11144/Javeriana.upsy14-2.mpmc 